PROCEEDINGS OF THE

AMERICAN MATHEMATICAL SOCIETY

Volume 127, Number 4, April 1999, Pages 1003-1010

S 0002-9939(99)04657-2

\title{
ON HYPERCYCLIC OPERATORS ON BANACH SPACES
}

\author{
LUIS BERNAL-GONZÁLEZ
}

(Communicated by David R. Larson)

\begin{abstract}
We provide in this paper a direct and constructive proof of the following fact: for a Banach space $X$ there are bounded linear operators having hypercyclic vectors if and only if $X$ is separable and $\operatorname{dim} X=\infty$. This is a special case of a recent result, which in turn solves a problem proposed by S. Rolewicz.
\end{abstract}

\section{INTRODUCTION}

In this paper $(X,\|\cdot\|)$ will denote a Banach space on $\mathbb{K}=\mathbb{R}$ (the real line) or $\mathbb{C}$ (the complex plane). $\mathbb{N}$ is the set of positive integers and $\mathbb{N}_{0}=\mathbb{N} \cup\{0\}$. Let $B(X)$ be the set of bounded linear operators from $X$ into itself. If $T \in B(X)$, then the orbit of a vector $x \in X$ is the set $\operatorname{Orb}(T, x)=\left\{T^{n} x: n \in \mathbb{N}_{0}\right\}$. A vector $x \in X$ is called hypercyclic for $T$ if $\operatorname{Orb}(T, x)$ is dense in $X$ or, in other words, there is no proper closed $T$-invariant subset of $X$ containing $x$. $T$ is called hypercyclic if it has a hypercyclic vector. Of course, if $X$ supports such an operator, then $X$ must be separable. In addition, $X$ must be infinite-dimensional; indeed, if $T^{n}$ is compact for some $n \in \mathbb{N}$, then $T$ is not hypercyclic ([Ki, Theorem 4.2]; see also [Ro]). A vector $x \in X$ is said to be cyclic (supercyclic) for an operator $T \in B(X)$ if the linear span of $\operatorname{Orb}(T, x)$ (if the set $\{\lambda y: y \in \operatorname{Orb}(T, x), \lambda \in \mathbb{K}\}$ ) is dense in $X$. An operator $T \in B(X)$ is cyclic (supercyclic) if it has a cyclic (a supercyclic) vector. It is evident that hypercyclicity implies supercyclicity and this, in turn, implies cyclicity. S. Rolewicz [Ro] was the first to isolate the concept of hypercyclicity. He showed in 1969 that on $l_{p}(1 \leq p \leq \infty)$ or $c_{0}$ there is a hypercyclic operator. He proved in fact that any multiple of the backward shift on Hilbert space by a scalar of modulus larger than one has hypercyclic vectors. Rolewicz also considered hypercyclicity on some Fréchet spaces.

An easy consequence of Baire's theorem is that a hypercyclic operator possesses a dense $G_{\delta}$ (so residual) set of hypercyclic vectors (see [Ki, Theorem 2.1] and [GeS]). In [GeS], R.M. Gethner and J.H. Shapiro studied hypercyclicity on Fréchet spaces and, as a consequence of a useful criterion ([GeS, Theorem 2.2]; see also [Ki, Theorem 1.4] and [Gr2]), obtained two classical theorems on entire functions; namely, Birkhoff's translation theorem [Bi] and MacLane's differentiation theorem [Ma]. In

Received by the editors May 29, 1997 and, in revised form, July 6, 1997.

1991 Mathematics Subject Classification. Primary 47A65; Secondary 47B37, 47B99.

Key words and phrases. Hypercyclic vector, linear operator, infinite-dimensional separable Banach space, biorthogonal system, backward weighted shift.

The author's research was supported in part by DGES grant \#PB93-0926 and the Junta de Andaluciá.

(C)1999 American Mathematical Society 
[GoS], G. Godefroy and J.H. Shapiro obtained several results about cyclicity of generalized backward shifts, supercyclicity of nonscalar adjoint multiplication operators, hypercyclicity of certain operators in the Fréchet space $H\left(\mathbb{C}^{n}\right)$ of entire functions on $\mathbb{C}^{n}$, and many more.

C. Read [Re] has constructed a Banach space operator such that every nonzero vector is hypercyclic. It is not known if such an example can exist on Hilbert space. B. Beauzamy [Be1], [Be2] and [Be3] constructed examples of linear operators on Hilbert spaces having dense, invariant linear manifolds all of whose nonzero elements are hypercyclic. P. S. Bourdon $[\mathrm{Bo}]$ has recently proved that any hypercyclic operator $T$ on a Banach space has a dense $T$-invariant linear manifold consisting, except for zero, entirely of hypercyclic vectors. I. Halperin, C. Kitai and P. Rosenthal $[\mathrm{HaKR}]$ showed that any linearly independent denumerable set of vectors in a separable infinite-dimensional Hilbert space is contained in the orbit of a hypercyclic vector. D.A. Herrero [He] proved that the algebra $B(H)$ of all bounded linear operators acting on a complex separable infinite-dimensional Hilbert space $H$ includes a large supply of chaotic (so hypercyclic) operators.

In 1969, S. Rolewicz [Ro, Problem 1] proposed the following problem: Does an infinite-dimensional separable Banach space support a hypercyclic operator? In [Hz], G. Herzog has characterized those (necessarily separable) Banach spaces $X$ which have supercyclic operators: $\operatorname{dim} X \in\{0,1,2\}$ or $\operatorname{dim} X=\infty$ if $\mathbb{K}=\mathbb{R}$, and $\operatorname{dim} X \in\{0,1\}$ or $\operatorname{dim} X=\infty$ if $\mathbb{K}=\mathbb{C}$. To prove this, he used methods of the theory of universal functions developed by K.G. Große-Erdmann [Gr1]. He also cleverly used a result due to R.I. Ovsepian and A. Pelczyński (see Theorem 2 below) about biorthogonal systems on Banach spaces. The operator furnished by G. Herzog in $[\mathrm{Hz}]$ is compact, so it cannot be hypercyclic. The answer to Rolewicz's problem has been recently given in the affirmative in [An2]. In fact, much more is proved there. A corollary of the main result in [An2] is that any infinite-dimensional Fréchet space admits a hypercyclic operator. The main result is demonstrated by introducing the concepts of $l^{1}$-complete space, locally $l^{1}$-convex space and quasiextension of $l^{1}$-operators, and then "translating" the problem to $l^{1}(\mathbb{N})$, for which H. N. Salas [Sa, Section 3] showed that identity plus a unilateral backward weighted shift with positive weights is hypercyclic. Nevertheless, we believe that a direct and constructive proof of the solution of Rolewicz's problem (at least for Banach spaces) can be of some interest for Banach analysts. Our technique will be similar to that used by H. N. Salas [Sa, Section 3]. Our result has been obtained independently of [An2].

To finish this section, we state the result to be proved:

Theorem 1. Let $X$ be an infinite-dimensional separable Banach space. Then there is $T \in B(X)$ such that $T$ is hypercyclic.

\section{Preliminary Results}

We will need two known facts for the proof of Theorem 1 . The first one can be found in $[\mathrm{OP}]$. The second one deals with Toeplitz matrices and is proved in the previously mentioned paper of Salas [Sa, Lemma 3.2].

Theorem 2. If $X$ is an infinite-dimensional separable Banach space, then there is a sequence $\left\{e_{p}\right\}_{0}^{\infty} \subset X$ and a sequence $\left\{\varphi_{q}\right\}_{0}^{\infty} \subset X^{*}$ with the following properties:

a) $\varphi_{q}\left(e_{p}\right)=\delta_{p q} \forall p, q \in \mathbb{N}_{0}$.

b) $\overline{\operatorname{span}\left\{e_{p}: p \in \mathbb{N}_{0}\right\}}=X$. 
c) If $\varphi_{q}(x)=0 \forall q \in \mathbb{N}_{0}$, then $x=0$.

d) $\left\|e_{p}\right\|=1 \forall p \in \mathbb{N}_{0}$ and $\sup _{q \in \mathbb{N}_{0}}\left\|\varphi_{q}\right\|=C<\infty$.

Lemma 3. Let $C_{n}=\left(c_{i j}(n)\right)$ be the $2^{k} \times 2^{k}$ matrix whose entries $c_{i j}(n)$ are the combinatory numbers $\left(\begin{array}{c}n \\ 2^{k}+j-i\end{array}\right)$. Let $B_{n}=\left(b_{i}(n)\right)$ be a column vector such that $b_{i}(n)$ is a polynomial in $n$ of degree at most $2^{k}-i\left(i=1,2, \ldots, 2^{k}\right)$. Then for $n$ large enough there is a solution $X_{n}=\left(x_{i}(n)\right)$ of the equation $B_{n}=C_{n} X_{n}$ and the entries $x_{i}(n)$ satisfy $\left|x_{i}(n)\right| \leq P / n^{i}$, where $P=P(k)$ is a constant.

\section{Proof of the Main Result}

We now prove Theorem 1. Fix a sequence $\left\{a_{p}\right\}_{1}^{\infty}$ of positive real numbers such that the series $\sum_{1}^{\infty} a_{p}$ converges. Define the self-mapping $S: X \rightarrow X$ by

$$
S x=\sum_{p=0}^{\infty} a_{p+1} \varphi_{p+1}(x) e_{p} \quad \forall x \in X
$$

where $\left\{e_{p}\right\}_{0}^{\infty}$ and $\left\{\varphi_{p}\right\}_{0}^{\infty}$ are furnished by Theorem 2. From property d) in that theorem, it follows that $S \in B(X)$ (in fact $\|S\| \leq C \sum_{1}^{\infty} a_{p}$ ). Let $I$ be the identity operator on $X$. We will show that the operator $T=I+S$ is hypercyclic.

From property b) in Theorem 2, we can select a dense sequence $\left\{z_{k}\right\}_{1}^{\infty}$ in $X$ of the form $z_{k}=\sum_{i=0}^{2^{k}-1} z_{i, k} e_{i}(k \in \mathbb{N})$ for suitable scalars $z_{i, k}\left(k \in \mathbb{N} ; i=0,1, \ldots, 2^{k}-1\right)$. We proceed by induction in order to construct an increasing sequence $\left\{n_{j}\right\}_{1}^{\infty}$ and vectors $y_{j}=\sum_{i=2^{j}}^{2^{j+1}-1} b_{i} e_{i} \in X(j \in \mathbb{N})$ satisfying

$$
\left\|y_{j}\right\| \leq 2^{-j}(1+\|T\|)^{-n_{j-1}}
$$

and

$$
\left\|T^{n_{j}}\left(\sum_{p=1}^{j} y_{p}\right)-z_{j}\right\| \leq 2^{-j} .
$$

Assume just for a moment that the integers $n_{j}$ and the vectors $y_{j}(j \in \mathbb{N})$ have been found. Define $y=\sum_{j=1}^{\infty} y_{j}$. We are going to show that $y$ is hypercyclic for $T$. Indeed, since $\left\{z_{k}\right\}$ is dense in $X$, it suffices to prove that $\lim _{k \rightarrow \infty}\left\|T^{n_{k}} y-z_{k}\right\|=0$. But this follows from (1), (2) and the next chain of inequalities:

$$
\begin{aligned}
\left\|T^{n_{k}} y-z_{k}\right\| & \leq\left\|T^{n_{k}}\left(\sum_{j=1}^{k} y_{j}\right)-z_{k}\right\|+\left\|\sum_{j=k+1}^{\infty} T^{n_{k}} y_{j}\right\| \\
& \leq 2^{-k}+\sum_{j=k+1}^{\infty}\left\|T^{n_{k}}\right\| \cdot\left\|y_{j}\right\| \\
& \leq 2^{-k}+\sum_{j=k+1}^{\infty} 2^{-j}(1+\|T\|)^{n_{k}}(1+\|T\|)^{-n_{j-1}} \leq 2^{-k+1} .
\end{aligned}
$$

Thus the theorem would be proved.

Firstly, observe that $T^{n}=\sum_{r=0}^{n}\left(\begin{array}{c}n \\ r\end{array}\right) S^{r}$, where $S^{0}=I$. Moreover, note that, by property a) in Theorem $2, S e_{i}=a_{i} e_{i-1}$ for $i \in \mathbb{N}$ and $S e_{0}=0$, so $S^{m} e_{s}=$ $\left(\prod_{l=s-m+1}^{s} a_{l}\right) e_{s-m}$ if $m \leq s$ and $=0$ otherwise, for each $m \in \mathbb{N}$. Let us try to find 
numbers $x_{1}, x_{2} \in \mathbb{K}$ and $n \in \mathbb{N}$ such that $\left\|T^{n}\left(x_{1} e_{2}+x_{2} e_{3}\right)-z_{1}\right\| \leq 1 / 2$. We have that

$$
\begin{aligned}
& T^{n}\left(x_{1} e_{2}+x_{2} e_{3}\right)-z_{1}=\left(x_{1}+n a_{3} x_{2}\right) e_{2}+x_{2} e_{3} \\
+ & {\left[\left(\begin{array}{l}
n \\
2
\end{array}\right) a_{1} a_{2} x_{1}+\left(\begin{array}{c}
n \\
3
\end{array}\right) a_{1} a_{2} a_{3} x_{2}-z_{0,1}\right] e_{0}+\left[\left(\begin{array}{c}
n \\
1
\end{array}\right) a_{2} x_{1}+\left(\begin{array}{c}
n \\
2
\end{array}\right) a_{2} a_{3} x_{2}-z_{1,1}\right] e_{1}=0 . }
\end{aligned}
$$

We now impose for both square brackets to be zero or, equivalently,

$$
\varphi_{p}\left(T^{n}\left(x_{1} e_{2}+x_{2} e_{3}\right)-z_{1}\right)=0 \quad(p=0,1) .
$$

Then we get the linear system

$$
\begin{gathered}
\left(\begin{array}{l}
n \\
2
\end{array}\right) x_{1}+\left(\begin{array}{l}
n \\
3
\end{array}\right) a_{3} x_{2}=\frac{z_{0,1}}{a_{1} a_{2}}, \\
\left(\begin{array}{c}
n \\
1
\end{array}\right) x_{1}+\left(\begin{array}{l}
n \\
2
\end{array}\right) a_{3} x_{2}=\frac{z_{1,1}}{a_{2}} .
\end{gathered}
$$

Its matrix with respect to $x_{1}, u_{2}$ (where $u_{2}=a_{3} x_{2}$ ) is the matrix $C_{n}$ of Lemma 3 for $k=1$. Then there is a constant $P$ such that $\left|x_{1}\right| \leq P / n$ and $\left|u_{2}\right| \leq P / n^{2}$ for $n$ large enough. Thus, we can take $n=n_{1} \in \mathbb{N}$ satisfying $\left\|x_{1} e_{2}+x_{2} e_{3}\right\| \leq 1 / 2$, $\left\|\left(x_{1}+n_{1} a_{3} x_{2}\right) e_{2}+x_{2} e_{3}\right\| \leq 1 / 2$ and, consequently, $\left\|T^{n_{1}}\left(x_{1} e_{2}+x_{2} e_{3}\right)-z_{1}\right\| \leq 1 / 2$, as required. Set $b_{2}=x_{1}, b_{3}=x_{2}$ and $y_{1}=b_{2} e_{2}+b_{3} e_{3}$. Then $\left\|y_{1}\right\| \leq(1 / 2)(1+\|T\|)^{-n_{0}}$ and $\left\|T^{n_{1}}\left(y_{1}\right)-z_{1}\right\| \leq 1 / 2$, where $n_{0}=0$. This completes the first step of the induction.

In order to make the process clearer, we also describe the second step of the induction. We now try to find numbers $x_{1}, x_{2}, x_{3}, x_{4} \in \mathbb{K}$ and $n \in \mathbb{N}$ such that

$$
\left\|T^{n}\left(y_{1}\right)+\sum_{i=1}^{4} x_{i} e_{4+i-1}-z_{2}\right\| \leq 1 / 4
$$

and

$$
\left\|\sum_{i=1}^{4} x_{i} e_{4+i-1}\right\| \leq(1 / 4)(1+\|T\|)^{-n_{1}} .
$$

With this we will define $y_{2}=\sum_{i=1}^{4} x_{i} e_{4+i-1}$. Again we want

$$
\varphi_{p}\left(T^{n}\left(y_{1}+y_{2}\right)-z_{2}\right)=0
$$

for $p=0,1,2,3$. Then

$$
\begin{gathered}
T^{n}\left(y_{1}+y_{2}\right)-z_{2}=x_{1} e_{4}+x_{2}\left[e_{5}+\left(\begin{array}{c}
n \\
1
\end{array}\right) a_{5} e_{4}\right]+x_{3}\left[e_{6}+\left(\begin{array}{c}
n \\
1
\end{array}\right) a_{6} e_{5}+\left(\begin{array}{l}
n \\
2
\end{array}\right) a_{6} a_{5} e_{4}\right] \\
+x_{4}\left[e_{7}+\left(\begin{array}{c}
n \\
1
\end{array}\right) a_{7} e_{6}+\left(\begin{array}{c}
n \\
2
\end{array}\right) a_{7} a_{6} e_{5}+\left(\begin{array}{c}
n \\
3
\end{array}\right) a_{7} a_{6} a_{5} e_{4}\right] .
\end{gathered}
$$

Thus

$$
\left\|T^{n}\left(y_{1}+y_{2}\right)-z_{2}\right\| \leq \sum_{i=1}^{4}\left|x_{i}\right|\left|Q_{i}(n)\right|
$$


where $Q_{i}(n)$ is a polynomial of degree $i-1$ in $n$. Condition (5) is equivalent to the system of linear equations

$$
\begin{aligned}
& \left(\begin{array}{l}
n \\
4
\end{array}\right) x_{1}+\left(\begin{array}{l}
n \\
5
\end{array}\right) a_{5} x_{2}+\left(\begin{array}{l}
n \\
6
\end{array}\right) a_{6} a_{5} x_{3}+\left(\begin{array}{l}
n \\
7
\end{array}\right) a_{7} a_{6} a_{5} x_{4}=d_{1}(n), \\
& \left(\begin{array}{l}
n \\
3
\end{array}\right) x_{1}+\left(\begin{array}{l}
n \\
4
\end{array}\right) a_{5} x_{2}+\left(\begin{array}{l}
n \\
5
\end{array}\right) a_{6} a_{5} x_{3}+\left(\begin{array}{l}
n \\
6
\end{array}\right) a_{7} a_{6} a_{5} x_{4}=d_{2}(n), \\
& \left(\begin{array}{l}
n \\
2
\end{array}\right) x_{1}+\left(\begin{array}{l}
n \\
3
\end{array}\right) a_{5} x_{2}+\left(\begin{array}{l}
n \\
4
\end{array}\right) a_{6} a_{5} x_{3}+\left(\begin{array}{l}
n \\
5
\end{array}\right) a_{7} a_{6} a_{5} x_{4}=d_{3}(n), \\
& \left(\begin{array}{l}
n \\
1
\end{array}\right) x_{1}+\left(\begin{array}{l}
n \\
2
\end{array}\right) a_{5} x_{2}+\left(\begin{array}{l}
n \\
3
\end{array}\right) a_{6} a_{5} x_{3}+\left(\begin{array}{l}
n \\
4
\end{array}\right) a_{7} a_{6} a_{5} x_{4}=d_{4}(n),
\end{aligned}
$$

where

$$
\begin{gathered}
d_{1}(n)=\left(1 / a_{1} a_{2} a_{3} a_{4}\right)\left[-b_{2}\left(\begin{array}{c}
n \\
2
\end{array}\right) a_{2} a_{1}-b_{3}\left(\begin{array}{l}
n \\
3
\end{array}\right) a_{3} a_{2} a_{1}-z_{0,2}\right] \\
d_{2}(n)=\left(1 / a_{2} a_{3} a_{4}\right)\left[-b_{2}\left(\begin{array}{c}
n \\
1
\end{array}\right) a_{2}-b_{3}\left(\begin{array}{c}
n \\
2
\end{array}\right) a_{3} a_{2}-z_{1,2}\right] \\
d_{3}(n)=\left(1 / a_{3} a_{4}\right)\left[-b_{2}-b_{3}\left(\begin{array}{c}
n \\
1
\end{array}\right) a_{3}-z_{2,2}\right] \\
d_{4}(n)=\left(1 / a_{4}\right)\left[-b_{3}-z_{3,2}\right] .
\end{gathered}
$$

Set $x_{1}=u_{1}, x_{2} a_{5}=u_{2}, x_{3} a_{6} a_{5}=u_{3}, x_{4} a_{7} a_{6} a_{5}=u_{4}$, and observe that $d_{i}(n)$ is a polynomial of degree $4-i$, thus Lemma 3 can be applied for $k=2$. Therefore $\left|u_{i}\right| \leq P / n^{i}$ and consequently $\left|x_{i}\right| \leq Q / n^{i}$ for another constant. By choosing $n$ large enough, we have that (4) is satisfied. Since we also have (5), it follows that (3) and (6) are also satisfied. Now set $x_{1}=b_{4}, x_{2}=b_{5}, x_{3}=b_{6}, x_{4}=b_{7}$ and we have $y_{2}=\sum_{i=4}^{7} b_{i} e_{i}$.

Define, for the sake of convenience, $b_{0}=b_{1}=0$. Assume now that $n_{0}=0<n_{1}<$ $\ldots<n_{k-1}$ and $y_{1}, \ldots, y_{k-1}$ have been chosen such that (1) and (2) hold. Consider the linear system

$$
\varphi_{p}\left(T^{n}\left(\sum_{j=1}^{k-1} y_{j}+\sum_{i=1}^{2^{k}} x_{i} e_{2^{k}+i-1}\right)-z_{k}\right)=0 \quad\left(p=0,1, \ldots, 2^{k}-1\right) .
$$

Observe that, for each $p \in\left\{0,1, \ldots, 2^{k}-1\right\}$, the left-hand side of (3) is

$$
\begin{aligned}
& \sum_{j=1}^{2^{k}} x_{j} \varphi_{p}\left(T^{n} e_{2^{k}+j-1}\right)-\varphi_{p}\left(z_{k}\right)+\sum_{j=1}^{k-1} \sum_{q=2^{j}}^{2^{j+1}-1} \varphi_{p}\left(T^{n} b_{q} e_{q}\right)=\sum_{j=1}^{2^{k}} x_{j}\left[\varphi_{p}\left(e_{2^{k}+j-1}\right)\right. \\
& \left.\quad+\sum_{q=1}^{n}\left(\begin{array}{l}
n \\
q
\end{array}\right) \varphi_{p}\left(S^{q} e_{2^{k}+j-1}\right)\right]-z_{p, k}+\sum_{q=2}^{2^{k}-1} b_{q}\left[\varphi_{p}\left(e_{q}\right)+\sum_{r=1}^{n}\left(\begin{array}{l}
n \\
r
\end{array}\right) \varphi_{p}\left(S^{r} e_{q}\right)\right]
\end{aligned}
$$

(use now that $p \leq 2^{k}-1$ and take $n \geq 2^{k+1}$ ) 


$$
\begin{aligned}
= & \sum_{j=1}^{2^{k}} x_{j} \sum_{q=1}^{2^{k}+j-1}\left(\begin{array}{l}
n \\
q
\end{array}\right)\left(\prod_{r=2^{k}+j-q}^{2^{k}+j-1} a_{r}\right) \cdot \varphi_{p}\left(e_{2^{k}+j-1-q}\right)-z_{p, k}+b_{p} \\
& +\sum_{q=2}^{2^{k}-1} b_{q} \sum_{r=1}^{q}\left(\begin{array}{l}
n \\
r
\end{array}\right)\left(\prod_{l=q-r+1}^{q} a_{l}\right) \delta_{p, q-r} \\
= & \sum_{j=1}^{2^{k}} x_{j}\left(\begin{array}{c}
n \\
2^{k}+j-1-p
\end{array}\right)\left(\prod_{r=p+1}^{2^{k}+j-1} a_{r}\right)-z_{p, k}+b_{p}+\alpha_{p},
\end{aligned}
$$

where $\alpha_{p}=0$ if $p=2^{k}-1$ and $\alpha_{p}=\sum_{q=p+1}^{2^{k}-1} b_{q}\left(\begin{array}{c}n \\ q-p\end{array}\right) \prod_{l=p+1}^{q} a_{l}$ if $p<2^{k}-1$. By putting $i=p+1$ for $i \in\left\{1, \ldots, 2^{k}\right\}$, the matrix of (3) with respect to $x_{1}, \ldots, x_{2^{k}}$ is the $2^{k} \times 2^{k}$ matrix with entries $d_{i j}=\left(\begin{array}{c}n \\ 2^{k}+j-i\end{array}\right) \cdot \prod_{r=i}^{2^{k}+j-1} a_{r}$. As shown in [Sa], the finite products of weights $a_{r}$ do not affect the estimation of solutions $x_{1}, \ldots, x_{2^{k}}$, so Lemma 3 can be applied. This is so because $\alpha_{p}\left(=\alpha_{i-1}\right)$ is a polynomial in $n$ of degree not greater than $\max \left\{q-i+1: q \leq 2^{k}-1\right\}=2^{k}-i$.

We conclude that if $n$ is large enough the solutions satisfy $\left|x_{i}\right| \leq P / n^{i}$, where $P=P(k)$ is a constant. We have arrived at the following:

$$
\begin{gathered}
T^{n}\left(\sum_{j=1}^{k-1} y_{j}+\sum_{i=1}^{2^{k}} x_{i} e_{2^{k}+i-1}\right)-z_{k}=\sum_{q=2}^{2^{k}-1} b_{q}\left(\sum_{j=0}^{n}\left(\begin{array}{l}
n \\
j
\end{array}\right) S^{j}\right) e_{q} \\
+\sum_{q=1}^{2^{k}}\left(\sum_{j=q}^{n}\left(\begin{array}{c}
n \\
j
\end{array}\right) S^{j}\right) x_{q} e_{2^{k}+q-1}-\sum_{q=0}^{2^{k}-1} z_{q, k} e_{q}+\sum_{q=1}^{2^{k}}\left(\sum_{j=0}^{q-1}\left(\begin{array}{c}
n \\
j
\end{array}\right) S^{j}\right) x_{q} e_{2^{k}+q-1} \\
=\sum_{q=1}^{2^{k}}\left(\sum_{j=0}^{q-1}\left(\begin{array}{c}
n \\
j
\end{array}\right) S^{j}\right) x_{q} e_{2^{k}+q-1},
\end{gathered}
$$

because, from (7), all coefficients in the $\left\{e_{p}\right\}_{0}^{\infty}$-expansion of the left-hand side are zero for $p=0,1, \ldots, 2^{k}-1$. Thus, from property $\mathrm{d}$ ) in Theorem 2 , it is derived that

$$
\left\|T^{n}\left(\sum_{j=1}^{k-1} y_{j}+\sum_{i=1}^{2^{k}} x_{i} e_{2^{k}+i-1}\right)-z_{k}\right\| \leq \sum_{q=1}^{2^{k}}\left(\sum_{j=0}^{q-1}\left(\begin{array}{c}
n \\
j
\end{array}\right)\|S\|^{j}\right)\left\|x_{q}\right\| \leq Q / n
$$

for another constant $Q=Q(k)$.

Choose $n_{k} \in \mathbb{N}$ large enough in such a way that $\left|x_{i}\left(n_{k}\right)\right| \leq 4^{-k}(1+\| T||)^{-n_{k-1}}$ $\left(i=1, \ldots, 2^{k}-1\right)$ and $n_{k}>\max \left\{2^{k} Q, n_{k-1}\right\}$. Set $x_{i}=b_{2^{k}+i-1}\left(i=1, \ldots, 2^{k}\right)$ and $2^{k+1}-1$

$y_{k}=\sum_{i=2^{k}}^{2^{k}} b_{i} e_{i}$. Then $\left\|y_{k}\right\| \leq 2^{-k}(1+\|T\|)^{-n_{k-1}}$ and $\left\|T^{n_{k}}\left(\sum_{j=1}^{k} y_{j}\right)-z_{k}\right\| \leq 2^{-k}$.

This is (1) and (2) for $j=k$. The theorem is now completely proved.

Corollary 4. Let $X$ be an infinite-dimensional separable Banach space and $\left\{e_{p}\right\}_{0}^{\infty}$, $\left\{\varphi_{q}\right\}_{0}^{\infty}$ sequences as in Theorem 2. Assume that $\left\{a_{p}\right\}_{1}^{\infty} \subset(0,+\infty)$ is a sequence 
satisfying $\sum_{1}^{\infty} a_{p}<\frac{1}{C}$. Then the operator $L \in B(X)$ given by

$$
L x=x+\sum_{n=1}^{\infty} \sum_{k=0}^{\infty}(-1)^{n}\left(\prod_{j=k+1}^{k+n} a_{j}\right) \varphi_{k+n}(x) e_{k}
$$

is hypercyclic.

Proof. If $T=I+S$ is the operator defined in the proof of Theorem 1 , then $\|S\|<1$, so $T$ is invertible. Then $T^{-1}$ is also hypercyclic (see $[\mathrm{Ki}]$ and $[\mathrm{HeK}]$ ). But $T^{-1}=$ $(I-(-S))^{-1}=I+\sum_{n=1}^{\infty}(-1)^{n} S^{n}=L$, and we are done.

\section{FinAL REMARKS AND OPEN PROBLEMS}

Note that the operator $T=I+S$ of Theorem 1 is a compact perturbation of identity, because $S$ is compact. Indeed, $S$ is a norm limit in $B(X)$ of a sequence of operators with finite-dimensional range. Property c) of $\left\{\varphi_{q}\right\}$ (Theorem 2) has not been used in our proof. G. Herzog does not use it either in the proof of his theorem on supercyclicity $[\mathrm{Hz}]$. We also remark that every power $T^{n}(n \in \mathbb{N})$ of our operator $T=I+S$ is also hypercyclic. This is due to a recent result of S. I. Ansari [An1]. To finish, we propose two up-to-date open problems:

1. What are the Banach spaces that admit hypercyclic operators not of the form $T=I+S$ with $S$ compact?

2. Is it possible to do in Banach spaces what Halperin, Kitai and Rosenthal did in Hilbert spaces: Given a denumerable set of vectors, find an operator and a vector whose orbit contains such a set?

\section{REFERENCES}

[An1] S. I. Ansari, Hypercyclic and cyclic vectors, J. Funct. Anal. 128 (1995), 374-383. MR 96h: 47002

[An2] S. I. Ansari, Hypercyclic operators on topological vectors spaces, J. Funct. Anal., to appear. CMP 98:01

[Be1] B. Beauzamy, Un opérateur sur l'espace de Hilbert, dont tous les polynômes sont hypercyclic, C. R. Acad. Sci. Paris, Sér. I Math. 303 (1986), 923-927. MR 88g:47010

[Be2] B. Beauzamy, An operator in a separable Hilbert space with many hupercyclic vectors, Studia Math. 87 (1987), 71-78. MR 89j:47004

[Be3] B. Beauzamy, An operator on a separable Hilbert space with all polynomial hypercyclic, Studia Math. 96 (1990)), 81-90. MR 91d:47004

[Bi] G. D. Birkhoff, Démonstration d'un théorème elémentaire sur les fonctions entières, C. R. Acad. Sci. Paris 189 (1929), 473-475.

[Bo] P. S. Bourdon, Invariant manifolds of hypercyclic vectors, Proc. Amer. Math. Soc. 118 (1993), 845-847. MR 93i:47002

[GeS] R. M. Gethner and J. H. Shapiro, Universal vectors for operators on spaces of holomorphic functions, Proc. Amer. Math. Soc. 100 (1987), 281-288. MR 88g:47060

[GoS] G. Godefroy and J. H. Shapiro, Operators with dense, invariant, cyclic vector manifolds, J. Funct. Anal. 98 (1991), 229-269. MR 92d:47029

[Gr1] K. G. Grosse-Erdmann, Holomorphe Monster und universelle Funktionen, Mitt. Math. Sem. Giessen 176, 1987. MR 88i:30060

[Gr2] K. G. Grosse-Erdmann, On the universal functions of G. R. MacLane, Complex Variables 15 (1990), 193-196. MR 91i:30021

[HaKR] I. Halperin, C. Kitai and P. Rosenthal, On orbits of linear operators, J. London Math. Soc. (2) 31 (1985), 561-565. MR 87e:47025

[He] D. A. Herrero, Hypercyclic operators and chaos, J. Operator Theory 28 (1992), 93-103. MR 95g:47031

[HeK] D. A. Herrero and C. Kitai, On invertible hypercyclic operators, Proc. Amer. Math. Soc. 116 (1992), 873-875. MR 93a:47023 
[Hz] G. Herzog, On linear operators having supercyclic vectors, Studia Math. 103 (3) (1992), 295-298. MR 93k:47033

[Ki] C. Kitai, Invariant closed sets for linear operators, Dissertation, University of Toronto, 1982 .

[Ma] G. R. MacLane, Sequences of derivatives and normal families, J. Analyse Math. 2 (1952), 72-87. MR 14:741d

[OP] R. I. Ovsepian and A. Pelczynski, The existence in every separable Banach space of a fundamental total and bounded biorthogonal sequence and related constructions of uniformly bounded orthogonal systems in $L^{2}$, Studia Math. 54 (1975), 149-155. MR 52:14941

[Re] C. Read, The invariant subspace problem for a class of Banach spaces, 2: Hypercyclic operators, Israel J. Math. 63 (1988), 1-40. MR 90b:47013

[Ro] S. Rolewicz, On orbits of elements, Studia Math. 32 (1969), 17-22. MR 39:3292

[Sa] H. N. Salas, Hypercyclic weighted shifts, Trans. Amer. Math. Soc. 347 (1995), 993-1003. MR 95e: 47042

Departamento de Análisis Matemático, Facultad de Matemáticas, Universidad de Sevilla, Apdo. 1160, Sevilla 41080, Spain

E-mail address: Ibernal@cica.es 